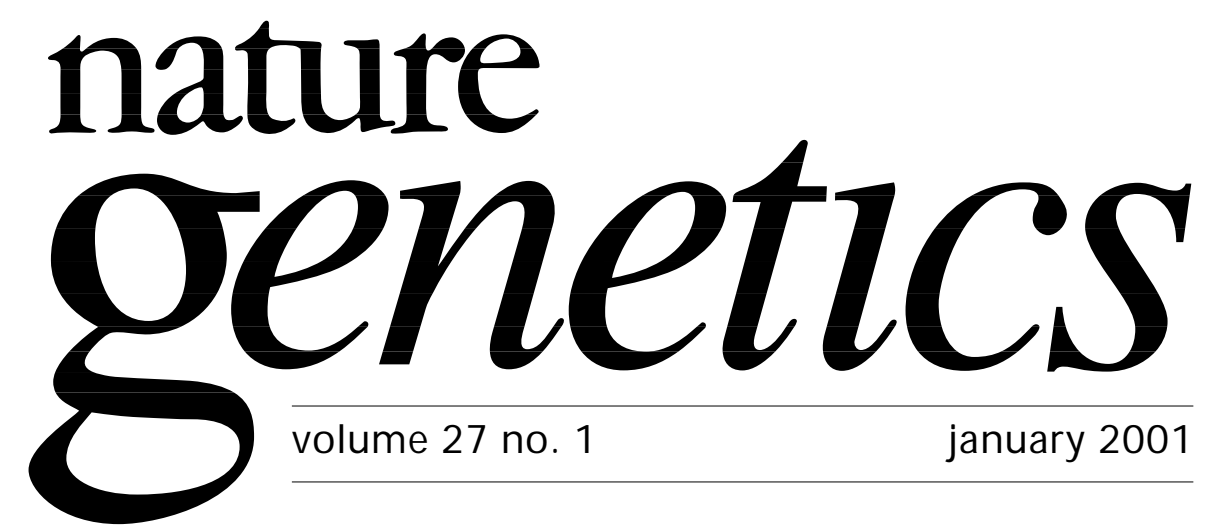

\title{
Chopping and changing
}

Accurate splicing of exons is critical to the molecular health of a cell. Testimony to this is the deleterious effects of thousands of splicing mutations that cause disease. A study presented by Adrian Krainer and colleagues ${ }^{1}$ (at Cold Spring Harbor Laboratory) on page 55 of this issue, however, indicates that human disease may be consequent to a much greater number of splicing mutations than is generally supposed. It suggests that many single base-pair mutations that are believed to act by terminating translation, for example, may instead disrupt splicing. As such, the study provides a new perspective in addition to challenging an old assumption.

The SR proteins, which are known to engage a range of RNA motifs called exonsplicing enhancers (ESEs), were discovered in the early 1990s (refs. 2-5). ESEs are found in exons, and in concert with the SR family of proteins, enhance the use of adjacent splice sites. Krainer and colleagues focused on one of the more potent splicing mutations of the tumor suppressor gene BRCA1, a transversion occuring six bases into exon 18 that results in exon skipping. Whereas the hypothesis that the mutation discrupts an ESE seems obvious, other experiments have indicated that ESEs may require a greater degree of disruption than that predicted by a single point mutation. And the identity of the ESE is 'fuzzy' - unlike transcription factors, members of the SR protein family are promiscuous in that they engage a range of different motifs.

Using scoring matrices constructed on the basis of previous studies ${ }^{6,7}$ of ESEs, they scanned the exon, and discovered the offending point mutation, encoding a transversion at position 6 , to fall within a motif with a high score (which indicates degree of similarity to known ESEs). Two other mutations were tested: an in-frame missense mutation that is predicted to disrupt the binding motif, and an in-frame nonsense mutation that lies outside of the binding motif. Splicing is disrupted in the former, but not the latter. As expained by Lynne Maquat (Univ. Rochester) on page 5 (ref. 8), these results indicate that any single-base change may exert deleterious effect by disrupting an ESE. The odds of this happening are higher than might be predicted, given the fuzziness of ESEs and that our knowledge of them is probably far from complete. Supporting this view is an additional finding of the study: of 50 additional point mutations that are have been demonstrated to disrupt splicing, about half disrupt high-score motifs.

This study is a timely reminder that splicing represents a major challenge to teasing apart gene expression. One may question the significance of an alternatively spliced isoform. Is it a product of happenstance - a fortuitous juxtaposition of nucleotides that happens to attract a protein that influences local splicing-or does its existence imply that it has a functional role? Is it translated? And, when function has been estab- 
1. Liu, H.-X., Cartegni, L., Zhang, M.Q. \& Krainer, A.R. Nature Genet. 27, 55-58 (2001).

2. Ge, H., Zuo, P. \& Manley, J.L. Cell 66 373-382 (1991)

3. Krainer, A.R., Conway, G.C. \& Kozak, D. Cell 66, 383-394 (1991)

4. Fu, X.D. \& Manaitis, T. Proc. Natl Acad. Sci. USA 89, 1725-1729 (1992)

5. Roth, M.B., Zahler, A.M. \& Stolk, J.A. J. Cell Biol. 115, 587-596 (1991).

6. Liu, H.-X., Xhang, M. \& Krainer, A.R. Genes Dev. 12, 1998-2012 (1998).

7. Liu, H.-X., Chew, S.L., Cartegni, L., Zhang, M.Q. \& Krainer, A.R. Mol. Cell. Biol. 20 1063-1071 (2000)

8. Maquat, L. Nature Genet. 27, 5-6 (2001).

9. Arch, R. et al. Science 257, 682-685 (1992).

9. Arch, R. et al. Science 257, $682-685(1992)$.
10. Xu, X. et al. Nature Genet. 22, 37-43 (1999).

11. Carstens, R.P., Wagner, E.J. \& Garcia-Blanco, M.A. Mol. Cell. Biol.20, 7388-7400 (2000). lished, how does one measure levels of the isoform? The presence of a 'spliceoform' can be detected by RT-PCR followed by sequencing or blotting, but this is a laborintensive approach and, with respect to quantification, crude at best.

A high-throughput method that is capable of differentially detecting and measuring levels of splice isoforms would be a welcome development, and not only with respect to having a means to determine what transcripts are spliced and when. One of the most useful applications of expression analysis is the simple but powerful use of profiles in diagnosis and prognosis. Superimposing a 'splice-assay' to query the presence of tumor-associated isoforms ${ }^{9-11}$ would probably heighten the degree of sensitivity of these molecular 'surveys', and provide researchers with a means of determining the general level of splicing activity in a cell. The control of splicing activity per se is not understood, but it seems plausible that the levels of splicing factors in the nucleus determine the rate (in addition to the type) of splicing, and hence the rate of export of mature transcripts into the cytoplasm.

The results of the current study provide insight into molecular mechanism. As genomic sequence is mined and new tools become available, we can look forward to similar studies and a clearer vision of the still largely-obscure world of the transcriptome.

\section{You say tomato, I say tomato}

Pronunciation is a moveable feast, spelling is less so. This month's issue of Nature Genetics is the first to subscribe to American English, rather than British English. Whereas Nature Genetics is an offshoot of the British journal Nature, with which it maintains close ties, it has been based in the United States since its launch in 1992. Nearly nine years on, and with six (soon to be seven) members of staff who are either North American or au fait with the spelling, the decision to adhere to American English is one that is based on practicality. There are three other new features in this issue. The Brief Communication now features a terse abstract so as to increase the odds that it will be picked up by electronic searches. In recognition of the fact that it is a report of primary research, dates of receipt and acceptance are now provided. And Acknowledgments of all research papers will include, should the authors so desire, a specification of who did what-so as to more clearly indicate the nature of their respective contributions. 